\title{
Can ultrasonic atomization generate nanometric droplets?
}

\author{
Santiago Jiménez, Antonio Lozano*, Esteban Calvo, José L. Santolaya, Félix Barreras \\ LIFTEC, CSIC - Universidad de Zaragoza, Spain \\ *Corresponding author: a.lozano@csic.es
}

\begin{abstract}
An ultrasonic wave packet propagating through a liquid medium is capable of generating a cloud of droplets emerging from its surface. A large number of experiments show that if the wave frequency is in the range of $1.5 \mathrm{MHz}-2.5 \mathrm{MHz}$, a major fraction of these droplets have a mean diameter of a few microns. Recently, some papers have been published claiming that the produced sprays also (or in some cases only) include a large number of nanometric droplets, in the framework of studies regarding the potential separation of ethanol-water mixtures by ultrasonic atomization. The presence of these ultrafine aerosols has been attributed to condensation of vapor on nuclei formed by cavitation. Based on some experimental results and a priori considerations regarding the measurement techniques applied in those works, this paper proposes a different nature and origin of these detected nanometric corpuscles; namely, that they are in fact particles corresponding to the solid residue left by the complete evaporation of the spray micrometric droplets, a well-established route for the formation of nanoparticles in virtually any kind of atomization technique..
\end{abstract}

\section{Keywords}

Ultrasonic atomization, sprays, droplets, nanoparticles.

\section{Introduction}

Since the first quarter of the past century [1] it is known that under certain conditions, an ultrasonic wave propagating on a liquid medium is capable of generating a cloud of droplets emerging from its surface. Spray formation depends on the nature of the liquid and the specific characteristics of the ultrasonic wave. In general, it is widely accepted that wave frequency essentially determines the droplet mean diameter, while the amplitude controls the flow rate [2]. It has also been demonstrated that atomization eventually ceases when increasing liquid viscosity [3]. This type of atomization is thought to be caused by two main mechanisms: cavitation inside the liquid mass, first proposed by Söllner in 1936 [4], and instabilities of capillary waves formed on its free surface. The relevance of each one of them might depend on the forcing frequency or the ultrasonic power [5]. Following the second theory, Lang proposed in 1962 his well-known expression relating ultrasonic vibration and droplet diameter [6]

$$
D_{n 0.5}=k\left(\frac{8 \pi \sigma}{\rho F^{2}}\right)^{1 / 3}
$$

where $D_{n 0.5}$ is the number median particle diameter, $\sigma$ is the surface tension coefficient, $\rho$ is the liquid density, $F$ is the forcing frequency and $k$ is an empirically determined constant whose value, according to Lang, was 0.34 . In spite of the fact that the theoretical justification of eq. (1) can be questioned [7] and that it completely neglects any possible role of cavitation, it is widely used, and provides predictions that sometimes are surprisingly accurate. 
When working with frequencies in the $\mathrm{MHz}$ range, sprays are generated with droplet diameters of the order of a few microns [2, 8-10]. Depending on the atomized liquid, larger droplets $(\sim 100 \mu \mathrm{m})$ can also be found and individually discerned by eye [11]. The micronsized ones are visible as a fine mist although they can be resolved if imaged with adequate magnification [2, 11]. In the last years, some papers have been published claiming that the produced sprays also include a large number of nanometric droplets. Yano et al. [12, 13] first detected them at the exit of an atomizing chamber with a $2.4 \mathrm{MHz}$ transducer applying Small Angle X-ray Scattering (SAXS). Kobara et al. [14] used a Differential Mobility Analyzer (DMA) and a Condensation Particle Counter (CPC) to measure the size distribution of these droplets atomizing water, ethanol and mixtures of both compounds also with a $2.4 \mathrm{MHz}$ piezoceramic disk. The nano-droplets would provide an explanation for a previous result by the same group showing an enrichment in alcohol of the mist with respect to the mixture being atomized [15] (a result that might be controversial according to other researchers [16]). More recently, Kudo et al. [17] using a similar sizing equipment analyzed sprays of both tap and pure water with forcing frequencies ranging from $200 \mathrm{kHz}$ to $2.4 \mathrm{MHz}$. The observed nanometric mode was attributed to condensation of vapors on nuclei formed by cavitation. In the present work, we wish to highlight some concerns in the interpretation of these results regarding the aerosol size distribution, and offer a simpler, alternative view: that these nanodroplets are actually nano-particles generated by evaporation of the micron-sized droplets. Two approaches are briefly presented: a priori considerations based on the techniques used and a series of experiments similar to those reported in the articles quoted above.

\section{A Priori Considerations}

SAXS is a non-invasive technique which indeed provided evidence of the presence of very fine $(1-100 \mathrm{~nm})$ corpuscles in the mist generated by an ultrasound atomizer $[12,13]$. It is, however, and to our knowledge, unable to characterize the state (liquid, solid) of that aerosol. In those works, Yano et al. were conducting the droplets to the measuring point dragging them with a strong $(10 \mathrm{l} / \mathrm{min}-50 \mathrm{l} / \mathrm{min})$ flow of air.

Earlier papers do not report the presence of such small droplets, probably because the sprays had not been analyzed with systems capable of measuring in the nanometric scale. For diameters down to $\sim 0.3 \mu \mathrm{m}$, size is usually determined by laser diffraction. For particles smaller than a micron, size is normally measured through electrostatic classification and optical counting. Two commercial combinations of DMA and CPC were used in the aforementioned works (TSI SMPS 3936 in [14], MSP WPS 1000XP in [17]). In both cases, a stream of air carried the aerosol generated in the atomizer to the inlet of the instrument, and then the droplets were size-classified in a DMA; finally each class was counted in a CPC. A key point in this process is the dilution of the primary aerosol line at the entrance of the DMA, typically in a ratio 1:5 to 1:10, with (usually) filtered, room air [18]. A cloud of fine mist is per se a dynamic, unstable system due to coagulation, coalescence, etc. In the case of tiny droplets, evaporation and condensation play a very relevant role, and one has to be very careful to maintain the conditions in the air stream and not to alter the droplet properties. In particular, the saturation ratio ( $>1$ due to the Kelvin effect) needed to 'preserve' the nanometric droplets of a particular diameter should be kept constant. Even assuming that this is feasible inside the atomization chamber and the tube connecting it to the DMA (which would imply, for instance, a very careful control of the temperature in the walls), the introduction of the so-called sheath air at the entrance of the classifier in the DMA necessarily implies a significant drop in vapor pressure. This in turn would provoke the rapid evaporation of the droplets. The lifetime of a $100 \mathrm{~nm}$ droplet at $20^{\circ} \mathrm{C}$ and $100 \%$ relative humidity is around $\sim 10^{-4} \mathrm{~s}$ [19], orders of magnitude below the residence time in the 
classifier. Similar arguments can be applied to the droplets being driven to the X-Ray beam path in the SAXS measurements, especially in the case of ethanol; typical lifetimes for $1 \mu \mathrm{m}$ water droplets in vapor-free environments are around $1 \mathrm{~ms}$. CPCs, operating either with butanol or water, also introduce significant changes in the flow carrying the classified aerosol, both in temperature and vapor pressure (e.g. [20, 21]); this, however, might be only a minor problem since even a complete evaporation would probably result in a nuclei on which later condensation would occur without major changes in the total number of measured droplets.

\section{Experiments}

In order to explicitly check the nature of the submicronic aerosol generated in an ultrasound atomizer, we designed a limited series of experiments, briefly described in what follows. Sprays were generated using commercial lead zirconate titanate PZT-4 piezoceramic disks from APC International Ltd. (Mackeyville, PA, USA), with a diameter of $20 \mathrm{~mm}$, and a thickness of $1.3 \mathrm{~mm}$. The main resonance frequency was measured to be $1.65 \mathrm{MHz}$, in agreement with the manufacturer's specifications. The disks were excited with a sinusoidal wave coincident with the resonance frequency, and variable amplitude. They were attached to the bottom plate of a vessel with a square section of $18 \mathrm{~cm} \times 18 \mathrm{~cm}$ and a height of 24 $\mathrm{cm}$. A relatively large section was chosen to avoid noticeable liquid heating from the piezoceramic transducer. The top plate of the vessel had an inlet and an outlet in opposite corners for the carrier gas flow $(1.5 \mathrm{lpm})$. A secondary line of filtered, dry air allowed a variable dilution of the flow exiting the chamber through a corrugated tube. Both flows were regulated with Bronkhorst Mass View MV 302 mass flow controllers. The voltage applied to the piezoceramic disks varied between $30 \mathrm{~V}$ and $50 \mathrm{~V}$. The atomized liquid was distilled water although in some cases, different amounts of $\mathrm{NaCl}$ were diluted before atomization.

Two different systems were employed to determine the aerosol size distribution. For the 0.5 $\mu \mathrm{m}-800 \mu \mathrm{m}$ range, a Malvern Mastersizer S (Malvern Panalytical Ltd., Malvern, UK) with a $300 \mathrm{~mm}$ focal length lens was used. Smaller diameters were measured with a Scanning Mobility Particle SizerTM (SMPSTM) Spectrometer from TSI (TSI Inc. Shoreview, MN, USA) composed by a 3080 Electrostatic classifier with a 3081 long-DMA, and a 3782 CPC. The latter absorbed $0.6 \mathrm{lpm}$ of the flow near the exit of the $25 \mathrm{~cm}$ long corrugated tube, driving it to the DMA; $3 \mathrm{Ipm}$ of sheath air were entrained there, which if seen as a mixture would result in a dilution ratio of 1:6. The SMPS provides number concentrations in the 14-700 nm range. The measurements from the Mastersizer are expressed in volume percentage. A sketch of the experimental setup is depicted in Figure 1.

\section{Results and Discussion}

Without any voltage, essentially no particles were detected at the SMPS, so no background subtraction was judged necessary. When voltage was supplied, a direct SMPS measurement of the mist distribution clearly detected a single peak around $100 \mathrm{~nm}$, in good agreement with the works quoted [12-14, 17]. The absolute concentration was similar to that reported by those researchers, and the position of the maximum laid somewhere between the results for pure and tap water in Kudo et al. [17].

When the mist exiting the chamber was diluted with filtered, dry air, the position of the peak remained roughly constant, whereas the concentration of the aerosol decreased nearly linearly with the dilution ratio, as shown in Figure 2 for the case of a disk voltage of $50 \mathrm{~V}$. At the same time, the mist at the exit of the corrugated tube, which was clearly visible by eye without dilution, disappeared when the dilution ratio exceeded $\sim 1: 2$. This mist, as reported in 
the past and explicitly shown below, consisted of water droplets $1 \mu \mathrm{m}-10 \mu \mathrm{m}$ in diameter, and its disappearance is a direct evidence of their evaporation in the progressively drier ambient. Even neglecting the subsequent dilution inside the DMA, this primary dilution would thus necessarily imply that any smaller droplet would completely evaporate.

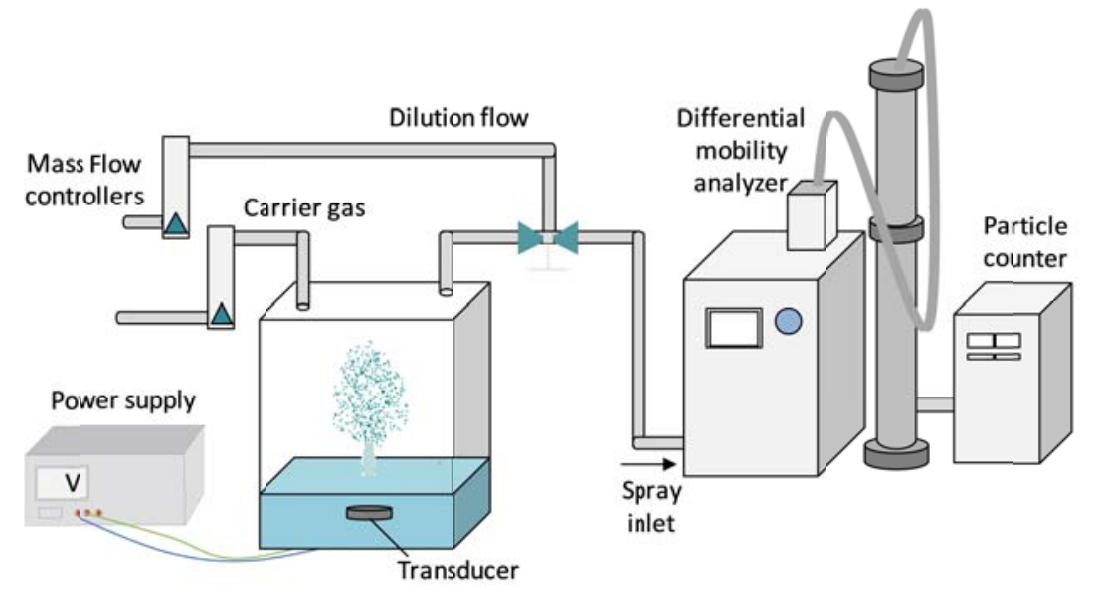

Figure 1. Scketch of the experimental setup.

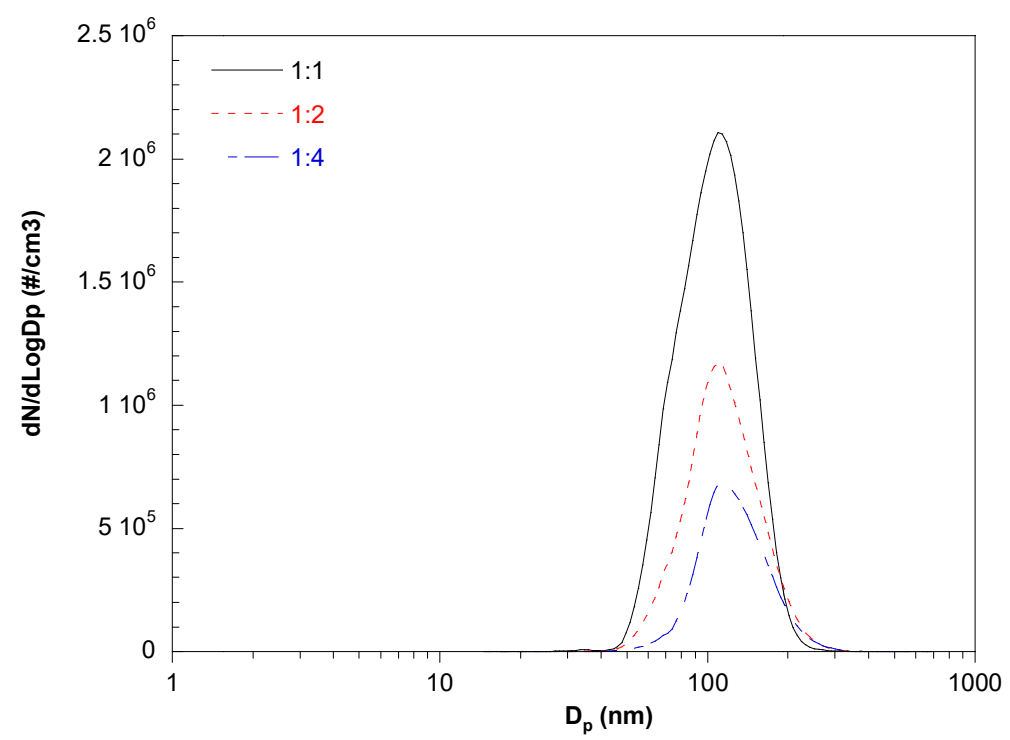

Figure 2. Aerosol size distribution measured with the SMPS at the exit of the tube carrying the air from the atomization chamber, with different dilution ratios with dry, filtered air (in the tube).

This result clearly shows that what is really being measured is the solid residue after complete droplet evaporation. There is, of course, a long experience in the generation of fine and ultrafine particles via atomization of liquids with variable amounts of solute and later evaporation of those droplets, and multiple commercial devices exist that are based on this principle (e.g. TOPAS ATM 226, Topas GmbH, Dresden Germany, or TSI 3097A). In fact, any kind of atomizer can be used for this purpose, and Kudo et al. referenced a few works in which ultrasound atomization was used in this way (e.g. [22]). Not having dissolved a product intentionally in this case, it is probably worth noting that quite small concentrations are sufficient to generate such fine particles: for instance, if a $5 \mu \mathrm{m}$ droplet originates a solid residue of $100 \mathrm{~nm}$, its non-volatile residue can be estimated around 8 ppm (for assumedly equal density of liquid and solid). 
To corroborate that the detected nanometric droplets are in fact the solid residue after evaporation, distilled water was replaced by a solution of $0.12 \mathrm{~g} / \mathrm{l} \mathrm{NaCl}$ in water. Increasing the non-volatile residue, the size of the particles should also increase. Considering a $\mathrm{NaCl}$ density of $2165 \mathrm{~kg} / \mathrm{l}$, the salt residue resulting from the complete evaporation of a $5 \mu \mathrm{m}$ droplet is a volume of $4.19 \times 10-21 \mathrm{~m} 3$. Assuming that it is forming a spherical particle, this volume corresponds to a $200 \mathrm{~nm}$ diameter sphere. The results measured with the DMA are displayed in Figure 3.

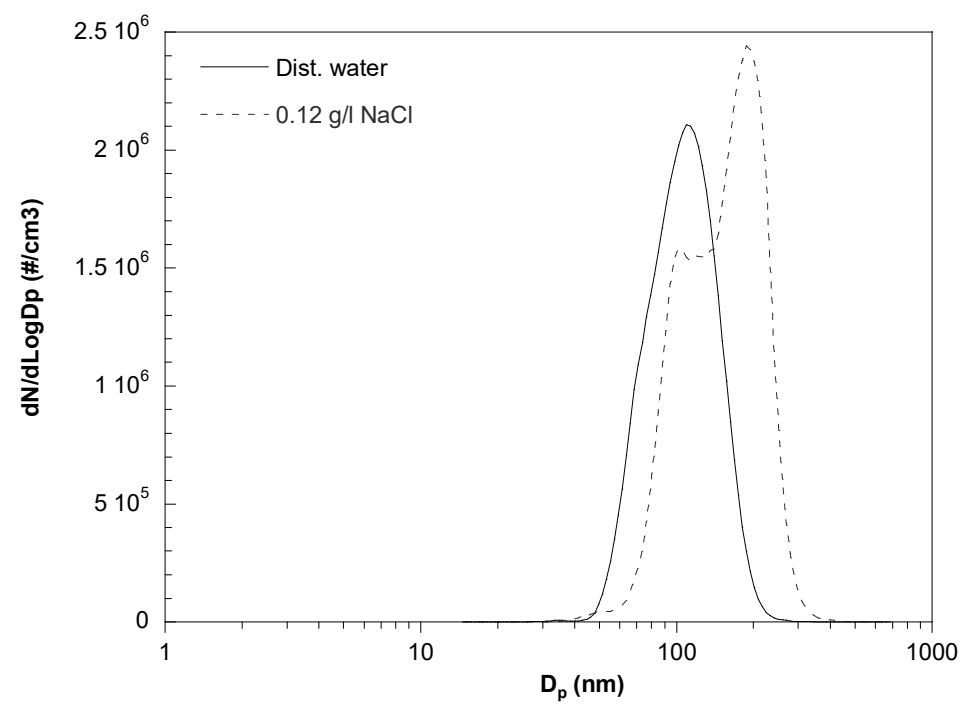

Figure 3. Fine aerosol size distribution measured with the SMPS at the exit of the atomizing chamber, working with distilled water and a salt solution.

As expected, the measured diameters shift towards higher values, with a maximum near 200 $\mathrm{nm}$. It is to be noted that if it is assumed that apart from the dissolved salt, the $5 \mu \mathrm{m}$ droplets also contain a non-volatile residue that would generate particles of $100 \mathrm{~nm}$, the combined effect of both solid residues would yield a spherical particle of $208 \mathrm{~nm}$, i.e. a negligible difference. A final check required ensuring that the addition of salt does not substantially alter the size of the mist droplets generated by ultrasonic atomization. To this end, measurements were performed with the laser diffractometer, dragging the droplets with a slow laminar carrier air flow. The disk transducer was forced with three different voltages, 30 $\mathrm{V}, 40 \mathrm{~V}$ and $50 \mathrm{~V}$. Results are presented in Figure 4. In good agreement with many previous papers, water spray droplet size distributions have a maximum around $5 \mu \mathrm{m}$. Addition of $\mathrm{NaCl}$ does not essentially modify the distributions. If anything, the peak is slightly displaced towards lower values, and the distributions widen with a higher percentage of smaller droplets. In any case, it seems fair to assume that with both liquids ultrasonic atomization produces a cloud of micronic droplets, with the peak near $5 \mu \mathrm{m}$.

Analyzing the results of these measurements, it seems clear that in the present experiments the nanometric objects measured with the DMA are actually nanoparticles product of the complete evaporation of the larger droplets generated by ultrasonic atomization. The question that arises is to what extent can it be claimed that ultrasonic atomization actually produces nanometric droplets. In their 2017 paper, Kudo et al. [17] acknowledge that the nanosized mist is probably not due to mechanophysical processes, such as cavitation and cleavage from capillary waves, and that these two mechanisms mainly contribute to generating microsized droplets. They assume that water vapor around the liquid column 
where the droplets are ejected is always supersaturated and that the nanosized mist is formed by vapor condensation on nuclei species supplied by cavitation. Larger sizes when operating with tap water compared to those for ultrapure water is justified by droplet coalescence. Their theory does not explain the conditions that determine the final size of the droplets formed by condensation. As in [14], evaporation in the chamber and/or measuring process was apparently not considered, although in both works a system very similar to the one implemented here was used (essentially identical in [14]). Nevertheless, all these results can also be explained in a simpler manner if it is supposed that what they are in fact measuring are the solid particles remaining after droplet evaporation. In particular, the difference in size when atomizing pure or tap water can easily be attributed to the difference in non-volatile residue in both liquids: the estimate made above for a $5 \mu \mathrm{m}$ droplet resulting in a $100 \mathrm{~nm}$ particle (for an $8 \mathrm{ppm}$ residue) gives roughly $30 \mathrm{~nm}$ for an initial non-volatile concentration of only $0.2 \mathrm{ppm}$ and $200 \mathrm{~nm}$ for $\sim 65 \mathrm{ppm}$.

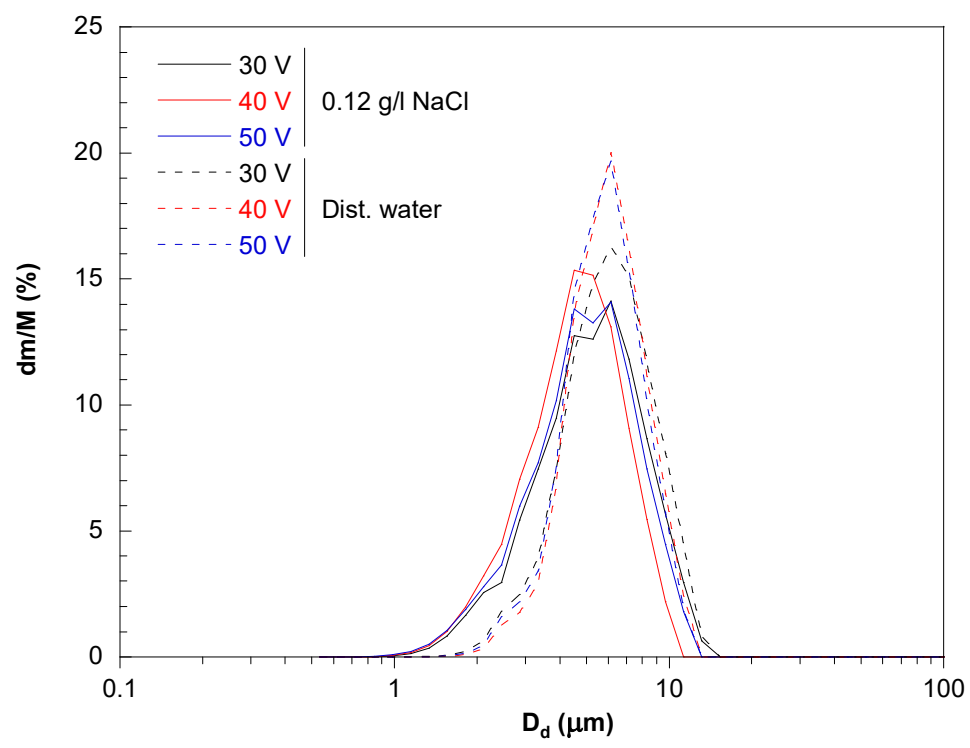

Figure 4. Size distributions of the droplets generated by the ultrasonic atomizer working with distilled water and salt water, as measured with the diffractometer.

Kobara et al. [14] argue that the nanometric droplets are actually produced by ultrasonic atomization and cannot derive from bigger ones because for ethanol concentrations higher than $20 \mathrm{~mol} \%$, they do not detect any micron-sized droplets. The latter observation might be however due to the fact that 'coarse' droplet production with ethanol is substantially lower than that with water, and the generated droplets might be already evaporated by the carrier gas stream in their experiments, which had a minimum flow rate of $15 \mathrm{l} / \mathrm{min}$. Ethanol ultrasonic atomization can indeed generate micrometric droplets that appear mixed with other larger ones $(\sim 100 \mu \mathrm{m})$ as reported in [11] and shown in Figure 5.

That there are micron sized droplets in the visible mist has been corroborated with Mastersizer measurements as shown in Figure 6. Two peaks are clearly observed in the size distribution expressed as volume percentage, corresponding to the micronic mist and the larger droplets. Their relative height are most likely incorrect, because the droplets were carried to the measuring point by an air dragging flow exiting from the top of the vessel, and probably the hundred-micron droplet concentration is grossly underestimated. 

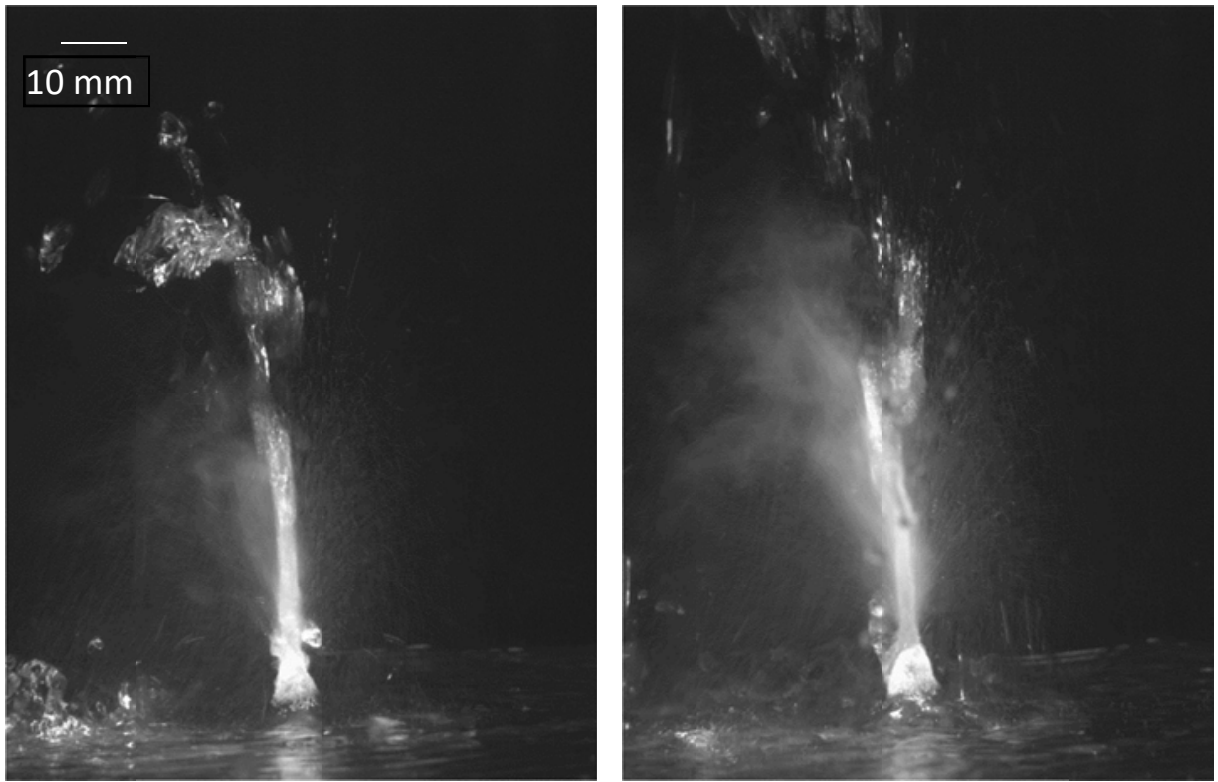

Figure 5. Spray produced by ultrasonic atomization of ethanol. Forcing voltages: $40 \mathrm{~V}$ (left) and $55 \mathrm{~V}$ (right)

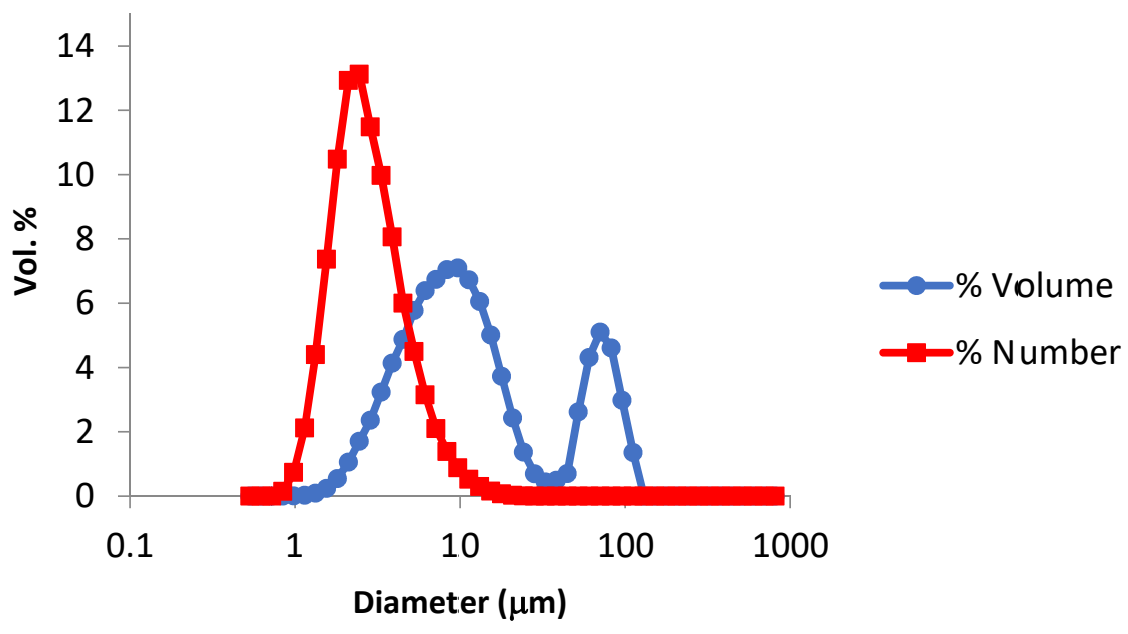

Figure 6. Doplet size distribution (in both liquid volume and droplet number percentages) of the ethanol spray in Figure 5

\section{Conclusions}

Experiments have been performed showing that nanometric particles can be obtained by complete evaporation of a micronic mist produced by ultrasonic atomization. Similar particles had been detected in previous works, but it had been claimed that they were nanometersized droplets directly generated by this type of atomization probably by deposition of liquid vapor on nuclei species ejected by the ultrasonic waves. Although this is a feasible process, we believe that our explanation is compatible with the results described in these papers, and is simpler and more plausible. The main reason for this opinion is that the method employed to measure the nanosized droplets, differential mobility analysis, normally requires a previous dilution of the aerosol samples, procedure that is accomplished mixing them with a sheath air flow that, unless otherwise specified, is entrained from the laboratory. This flow can be expected not to be saturated, and will evaporate droplets of such small diameters leaving only the non-volatile solid residue. Effectively, the size of the measured nanometric 
particles is congruent with this explanation. Caution should be exercised when assuming that ultrasonic atomization does produce nanometric droplets. The very well established generation of nano-particles by droplet evaporation should be carefully analyzed before discarding it

\section{Acknowledgments}

Partial support of the Regional Government of Aragon to the "Fluid Mechanics for a Clean Energy" Research Group (T01_20R) is acknowledged.

\section{References}

[1] Wood, W.R. and Loomis, A.L., The physical and biological effects of high frequency sound-waves of great intensity, Philosophical Magazine, 4, 22, 417-437, 1927.

[2] Barreras, F., Amaveda, H., Lozano, A., Transient High-Frequency Ultrasonic Water Atomization, Experiments in Fluids, 33, 3, 405-413, 2002.

[3] Lozano, A., García, J.A., Navarro, J.L., Calvo, E., Barreras, F., Influence of viscosity on droplet size distribution and generation rate in ultrasonic atomization Atomization and Sprays, 20, 11, 923-934, 2010.

[4] Söllner, K., Experiments to demonstrate cavitation caused by ultrasonic waves, Transactions of the. Faraday Society, 32, 1537-1538, 1936.

[5] Simon, J.C., Sapozhnikov, O.A., Khokhlova, V.A., Crum, L.A., Baile, M.R., Ultrasonic atomization of liquids in drop-chain acoustic fountains, Journal of Fluid Mechanics, 766, 129-146, 2015.

[6] Lang, R.J., Ultrasonic Atomization of Liquids, Journal of the Acoustic. Society of America, 34, no. 1, 6-8, 1962.

[7] Qi, A., Leslie Y. Yeo, L.Y., Friend, J.R., Interfacial destabilization and atomization driven by surface acoustic waves, Physics of Fluids, 20, 074103 1-14, 2008.

[8] Donnelly T.D., Hogan J., Mugler A., Schommer, N., Schubmehl, M., Bernoff, A.J., Forrest, B., An experimental study of micron-scale droplet aerosols produced via ultrasonic atomization, Physics of Fluids, 16, 8, 2843-2851, 2004.

[9] Yasuda, K., Bando, Y., Yamaguchi, S., Nakamura, M., Oda, A., Kawase, Y., Analysis of concentration characteristics in ultrasonic atomization by droplet diameter distribution, Ultrasonics Sonochemistry, 12, 37-41, 2005.

[10] Chiba, C., Atomization of liquid by immersed and convergent ultrasonic vibrators, Bulletin of the Japanese Society of Mechanical Engineering, 18, 118, 376-382, 1975.

[11] Lozano, A., García, J.A., Alconchel, J., Barreras, F., Calvo, E., Santolaya, J.L., Ultrasonic atomization of alkanes and alcohols, Atomization and Sprays, 27, 10, 875-891 2017.

[12] Yano, Y.F., Matsuura, K., Fukazu, T., Abe, F., Wakisaka, A., Kobara, H., Kaneko, K., Kumagai, A., Katsuya, Y., Okui, M., Tanaka, M., Small-angle X-ray scattering investigation of water droplets in mist, Journal of Applied Crystallography, 40, s318-s320, 2007.

[13] Yano, Y.F., Matsuura, K., Fukazu, T., Abe, F., Wakisaka, A., Kobara, H., Kaneko, K., Kumagai, A., Katsuya, Tanaka, M., Small-angle x-ray scattering measurement of a mist of ethanol nanodroplets: An approach to understanding ultrasonic separation of ethanol-water mixtures, The Journal of Chemical Physics, 127, 031101, 2007.

[14] Kobara, H., Tamiya, M., Wakisaka, A., Fukazu, T., Matsuura, K., Relationship Between the Size of Mist Droplets and Ethanol Condensation Efficiency at Ultrasonic Atomization on Ethanol-Water Mixtures, AIChE Journal, 56, 3, 810-814, 2010.

[15] Matsuura, M., Fujii, K., Sato, T., Ethanol separation from ethanol-water solution by ultrasonic atomization and its proposed mechanism based on parametric decay instability of capillary wave, Journal of Chemical Physics, 114, 2382, 2011.

[16] Spotar, S., Rahman, A., Gee, O.C., Jun, K.K. and Manickam, S., A revisit to the separation of ehatnol-water using ultrasonic distillation as a separation process, Chemical Engineering and Processing, 87, 45-50, 2015. 
[17] Kudo, T., Kazuhiko Sekiguchi, K., Sankoda, K., Namiki, N., Nii, S., Effect of ultrasonic frequency on size distributions of nanosized mist generated by ultrasonic atomization, Ultrasonics Sonochemistry, 37, 16-22, 2017.

[18] Flagan, R.C., Differential Mobility Analysis of Aerosols: A Tutorial, KONA Powder and Particle Journal, 26, 254-268, 2008.

[19] Hinds, W.C., Aerosol Technology, John Wiley \& Sons, NY, USA, 1982.

[20] Sem, G.J., Design and performance characteristics of three continuous-flow condensation particle counters: a summary, Atmospheric Research, 62, 267-294, 2002.

[21] Hering, S., Stolzenburg, M.R., Quant, F.R., Oberreit, D.R. and Ready, P.B., A laminarflow, water-based condensation particle counter (WCPC), Aerosol Science and Technology, 39, 659-672, 2005.

[22] Nii, S., Oka, N., Size-selective separation of submicron particles in suspensions with ultrasonic atomization, Ultrasonics Sonochemistry, 21, 2032-2036, 2014. 